\title{
Teacher training model - towards a Collaborative Rubric Bank
}

\author{
Julia Espinoza-Guzmán \\ TEC Digital \\ Tecnológico de Costa Rica \\ Email: juliaespinoza@itcr.ac.cr
}

\author{
Pablo Gamboa-Camacho \\ Depto. Admisión y Registro \\ Tecnológico de Costa Rica \\ Email:pgamboa@itcr.ac.cr
}

\author{
Mario Chacón-Rivas \\ TEC Digital \\ Tecnológico de Costa Rica \\ Email: machacon@itcr.ac.cr
}

\begin{abstract}
Resumen-Beyond providing alternatives to build rubrics, a group of researchers in Tecnológico de Costa Rica found the need to implement best practices in the definition and validation of evaluation rubrics, to encourage the creation of a Higher Education bank of assessment instruments. To achieve this goal, in the Tecnológico de Costa Rica, a teacher training process was established, which includes cooperative-collaborative work, scaffolding for the construction of knowledge and assessment tests carried out in the institutional LMS. The ultimate purpose is to build skills in teachers to design more robust rubrics and thus to form an institutional bank of evaluation instruments to share among colleagues. The first results meet the expectations established for the training model, as well as those of the teachers who participate in the training and use the rubrics for evaluation in their courses, as shown in the paper.
\end{abstract}

Index Terms-Assessment rubric, e-learning, ICT, LMS, bank of rubrics

\section{INTRODUCCIÓN}

En educación superior se identifican múltiples experiencias relacionadas con uso de rúbricas en procesos de evaluación [1] [2] y uso desde plataformas digitales [3]. Además, se identifica una estructura base para su construcción y mejores prácticas para su uso [4]. Sin embargo, el aprovechamiento de esta herramienta dependerá de la metodología didáctica, software de apoyo, acompañamiento de expertos, así como de características propias de la institución y su entorno. Ya que como indica [5] "Los programas de desarrollo de la facultad pasan por alto a menudo los factores que promueven o inhiben el uso de tecnologías entre profesores." Entre estos factores están la motivación para usar el LMS, falta de programas de formación enfocados en las necesidades docentes y en lograr un mejor dominio de la tecnología, su uso en la docencia [5] y la falta de tiempo [6]. En este mismo sentido, [6] citando a [7] señala factores internos a los docentes que, también, representan barreras tales como falta de confianza, la resistencia al cambio, la percepción de que el uso de las tecnologías "no produce realmente beneficios".

En el Instituto Tecnológico de Costa Rica (TEC), la Unidad TEC Digital desarrolla software de apoyo a la enseñanza y aprendizaje, que se incluye como un servicio agregado dentro del LMS, este último soportado en tecnología dotLRN. En el 2013, se liberó una versión de la aplicación denominada Administrador de Rúbricas que permite crear, publicar, compartir, asociar y realizar evaluaciones por medio de rúbricas creadas en la plataforma [8].

Luego del lanzamiento de la herramienta tecnológica, durante los dos años siguientes, el esfuerzo de capacitación se centró en la promoción del uso de rúbricas, en la creación considerando buenas prácticas y en el aprovechamiento de las funcionalidades que ofrece [8]. A partir del año 2015, se reorienta el modelo de capacitación con el objetivo de promover el mejoramiento del proceso de creación y uso de rúbricas bajo un enfoque cooperativo-colaborativo, aprovechando las condiciones del LMS y la versión actualizada del Administrador de Rúbricas.

A continuación se presenta el marco teórico, trabajos relacionados, el enfoque de formación docente propuesto, resultados obtenidos a la fecha, así como conclusiones y trabajo futuro establecido.

\section{MARCO TEÓRICO Y TRABAJO RELACIONADO}

Se identifican múltiples experiencias relacionadas con uso de rúbricas en procesos de evaluación en centros de enseñanza superior [1], [2], así mismo se distintos autores presentan una estructura base y mejores prácticas para la creación de estas [4] y [9].

De acuerdo con [10] las rúbricas de evaluación permiten "clarificar el alcance del proceso formativo de los estudiantes" ya que como indican [3] "... en el ámbito de la educación superior, la rúbrica se convierte tanto en una estrategia válida para la orientación y seguimiento del trabajo del alumnado, con identidad suficiente y autónoma al servicio de un determinado proceso formativo; como en una escala de valoración asociada a la evaluación, con entidad propia o bien, al servicio de otros instrumentos como puede ser el portafolio. De este modo, la rúbrica se presenta como un recurso para la evaluación integral y formativa, como un instrumento de orientación o como herramienta pedagógica.“

Desde esta perspectiva es que se valora el impacto de las rúbricas en la educación superior. Ahora bien, existen procesos recomendados y probados para construir rúbricas de evaluación [4], [9] y [11] que muestran el paso a paso para obtener este tipo de instrumentos de evaluación. 
En cuanto a la construcción cooperativa-colaborativa de rúbricas se registran experiencias en educación superior con un abordaje conjunto para generar una escala de evaluación que considere la perspectiva y expectativas de docente y estudiantes [12], [13] y [11].

Inicialmente en el TEC, para impulsar la creación de rúbricas y el uso de la aplicación en el LMS se ofrecieron cursos de 8 a 12 horas de duración; el trabajo consistió en aplicar el proceso para crear rúbricas, conocer el uso de la aplicación integrada al LMS y hacer un ejercicio de cómo evaluar usando la rúbrica. Aunque bajo esta modalidad se generaron muchos instrumentos de evaluación, no todos los profesores lograron dar el paso hacia la incorporación de su rúbrica en su práctica docente, y por limitaciones de tiempo tampoco había oportunidad de que el facilitador de la capacitación validara todas las rúbricas creadas.

Para solventar esta situación, se evaluaron opciones para reforzar la formación docente en el sentido de crear rúbricas más robustas y sobre todo que sean utilizadas para aprovechar las ventajas que ofrecen para estudiantes y profesores [14] y [3]. Entonces se hizo un nuevo planteamiento de formación que incluye aspectos como aprendizaje cooperativo-colaborativo, andamiaje, aprender a aprender y recomendaciones para facilitar el uso de LMS entre docentes. A continuación se describe el contexto en que son considerados estos aspectos.

\section{II-A. Aprendizaje Cooperativo-Colaborativo:}

Se promueven procesos de colaboración entre pares [10], y en este mismo sentido, [15] señala que "Con frecuencia, los alumnos pueden explicar muy bien las ideas difíciles a sus compañeros, ya que saben traducir el discurso del docente a su propio lenguaje.” Para el caso que nos ocupa, los participantes asumen el rol de aprendices, entonces colaboran y valoran el trabajo de los compañeros, para esto cuentan con guías de trabajo que promueven la interacción para el aprendizaje, la reflexión y evaluación de las actividades de aprendizaje [16].

\section{II-B. Banco de Rúbricas:}

En este concepto se considera la creación de un catálogo de rúbricas de evaluación que pueden ser la base para utilizarlas o adaptarlas a nuevos contextos; en el caso específico, debe responder a un proceso definido para la creación, revisión y validación de rúbricas orientadas a la Educación Superior, así como un proceso de publicación y puesta a disposición de la comunidad docente por medio del LMS institucional.

\section{II-C. Aprender a aprender:}

En la propuesta de formación se contempla lo que expone [17] en el sentido de transitar por "la asimilación, reflexión e interiorización"que permiten al estudiante "valorar y profundizar las distintas situaciones vitales en las que tiene que tomar una opción personal. Existe, pues, un proceso reflexivo, ya que se trata de una incorporación consciente y responsable de los hechos, conceptos, situaciones, experiencias, que implica aceptar el aprendizaje desde la perspectiva del alumno y relacionado con ámbitos específicos. Por lo tanto, se trata de un aprendizaje para desarrollar la actitud crítica y la capacidad de toma de decisiones. Estas dos características definen el proceso de aprender a aprender."

\section{ENFOQUE PROPUESTO}

Como se menciona anteriormente, se plantea una propuesta de formación docente que fortalece las capacidades para desarrollar rúbricas robustas a través de acciones cooperativascolaborativas entre expertos y colegas, con un objetivo secundario y de más largo plazo para la creación de un grupo o banco de rúbricas de evaluación revisadas y disponibles para futuros procesos de evaluación. La propuesta incluye fases, guías de trabajo que aplican los componentes clave descritos anteriormente, con el propósito de crear capacidades en los docentes en relación con las rúbricas como instrumento para evaluar.

\section{III-A. Componentes clave de la propuesta}

Estrategia que contempla acciones como:

1. Conformación de un equipo de especialistas institucionales en Evaluación (una doctora en Educación y experta en Evaluación, un Psicopedagogo, un Doctor en Computación, un Ingeniero en Computación y una máster en Tecnología educativa, todos docentes).

2. Formulación del proceso de formación docente que propicie el aprendizaje cooperativo-colaborativo, el aprender a aprender, andamiaje y buenas prácticas.

3. Utilización de técnicas para fomentar el uso del LMS, como uso progresivo de herramientas con el objetivo de crear confianza en los participantes y que valore las aplicaciones disponibles para su práctica docente.

4. Creación de recursos didácticos enfocados en explicar las bondades de las rúbricas ${ }^{1}$, el proceso para su construcción, buenas prácticas y ejemplos desarrollados, experiencias reales de profesores ${ }^{2}$ del TEC de tal forma que los participantes en las capacitaciones conozcan casos concretos aplicados en la misma universidad.

5. Proceso de formación docente organizado en sesiones a lo largo de 5 semanas, con una duración total de 25 horas, en que se utilizan diversas herramientas del LMS institucional como el Gestor de Actividades de Aprendizaje, el gestor de evaluaciones y el de Rúbricas. Eso permite a los participantes conocer y explorar las herramientas y vivir la experiencia relacionada con rúbricas desde la perspectiva de los estudiantes.

III-B. Propuesta de formación cooperativa-colaborativa entre docentes para crear el banco de rúbricas

A partir de 2015 se puso en práctica la formación docente que contempla los aspectos clave mencionados. A continuación se encuentra la descripción de cada sesión de trabajo de la capacitación.

Sesión introductoria: incluye una descripción del curso, metodología de trabajo, configuración del perfil personal en la

\footnotetext{
${ }^{1}$ https://www.youtube.com/watch?v=sHgLed $87 \mathrm{xf} 8$

${ }^{2}$ www.youtube.com/embed/Cmq8naft_aY
} 
plataforma LMS, diagnóstico de conocimientos previos sobre rúbricas y foro de presentación de los participantes.

Sesión 1: presenta el marco teórico sobre rúbricas, ventajas y limitaciones. Exposición de casos de profesores del TEC que usan rúbricas como una forma de conectar con la realidad cercana.

Sesión 2: a partir de las especificaciones del trabajo para el cual hará la rúbrica, cada particpante, aplica el procedimiento para construir la rúbrica de evaluación [4] y [9]. Se facilita una plantilla de trabajo, videos explicativos, infografías y otros recursos para el aprendizaje. Adicionalmente, se proporcionan recursos con ejemplos y contraejemplos de rúbricas para que el profesor revise buenas prácticas y aquellas que pueden inducir a error. Esta actividad es individual, con la guía de los facilidadores de la formación, el producto es la primera versión de la rúbrica.

Sesión 3: en grupo los participantes reciben los siguientes documentos: enunciado de un trabajo y su rúbrica asociada, así como la guía de trabajo cooperativo-colaborativo con un conjunto de preguntas que permiten identificar puntos fuertes y debilidades en una rúbrica determinada. El trabajo es cooperativo-colaborativo entre pares. A cada participante se le insta a reflexionar para que, a partir de la guía de trabajo, detecte áreas de mejora en la primera versión de su propia rúbrica. El facilitador promueve la evaluación de 360 grados en el sentido de hacer autoevaluación, co-evaluación y evaluación por parte del docente.

Sesión 4: consiste en registrar la rúbrica en el LMS como un recursos docente, por lo que puede ser utilizada en distintos cursos. Intencionalmente este paso se realiza cuando la rúbrica está finalizada en un medio físico o digital, con lo que se pretende bajar la carga cognitiva de aplicar el proceso de elaboración de rúbricas y a la vez aprender a usar la herramienta tecnológica. Para calificar el producto del trabajo de esta actividad el facilitador de la capacitación utiliza una "Rúbrica para calificar Rúbricas" registrada en el LMS.

Sesión 5: explora la evaluación con la rúbrica desde el LMS y considera actividades como:

- Realizar prueba de evaluación con un espacio de simulación semejante a un curso, con estudiantes ficticios. Permite valorar si la rúbrica es apropiada para sus expectativas.

- Valorar los indicadores de rendimiento obtenido por los estudiantes al ser evaluados con la rúbrica. Esta información puede ser usada por el profesor para valorar los 5 factores que detallan [14] y [18] desde la perspectiva del estudiante y del profesor.

- Exportar la calificación realizada como evidencia para procesos de acreditación de carreras.

Con los participantes se enfatiza que, si bien es cierto construir una rúbrica implica horas de trabajo un beneficio se refleja al calificar con este instrumento, y que su uso desde el LMS reduce hasta un $40 \%$ de tiempo en la calificación y devolución de resultados a los estudiantes [14]. En este contexto los facilitadores aprovechan este indicador para promover el uso de tecnologías y la importancia de aprender
Cuadro I

ESTADÍSTICAS DE USO DE RÚBRICAS EN 2014 Y 2017

\begin{tabular}{lrr}
\hline Descripción & 2014 & 2017 \\
\hline Cantidad de rúbricas creadas & 729 & 1402 \\
Promedio de rúbricas por autor & 3,64 & 3,65 \\
Cantidad de autores & 200 & 384 \\
Cursos o comunidades que utilizan rúbricas & 152 & 1376 \\
\hline \multicolumn{2}{c}{ Fuente: TEC Digital }
\end{tabular}

nuevas funcionalidades del LMS como una forma de romper las barreras que reporta [6] respecto a la falta de tiempo de los docentes y el sentido de que no producen beneficios reales.

\section{RESUltados A LA FECHA}

La aplicación de rúbicas está disponible para los profesores desde finales del año 2013. Se cuenta con registro de creación de rúbricas y talleres de formación desde ese año a la fecha actual.

En el cuadro I se presentan algunas estadísticas del crecimiento en el uso de las rúbricas por parte de los docentes.

En cinco cursos que se han ofrecido entre enero 2016 y mayo 2017 se tienen los siguientes resultados:

1. Se capacitaron 89 docentes, de los cuales 82 profesores aprobaron el curso de Rúbricas y siete profesores perdieron el curso, que indica un $92.13 \%$ de aprobación.

2. En una encuesta que se aplicó, los participantes en la capacitación registraron que durante el proceso de formación y especialmente en las etapas que se hace reflexión encuentran formas de revisar su práctica docente para revisar especificaciones de la asignación y sobre la forma de evaluar. Un hallazgo relevante es que al analizar los resultados de la calificación con la rúbrica y tomando en cuenta los resultados que muestran los gráficos de la aplicación en el LMS, los profesores notan la necesidad de reforzar temas en clase como una forma de mejorar el desempeño de los estudiantes.

3. Entre 2014 y 2017, se mantiene el promedio de rúbricas por autor según consta en el Cuadro I. El hecho relevante, en este caso, es que el uso de estos instrumentos de evaluación se ha incrementado en $800 \%$ de cursos en el TEC en ese mismo periodo.

4. Se aplicó un cuestionario a dos grupos de capacitación con el objetivo de validar la metodología de enseñanzaaprendizaje utilizada y las posibilidades de compartir y reutilizar rúbricas. En el cuadro II se presentan los resultados principales.

De acuerdo con los resultados de las encuestas, aplicadas a los participantes, la mayoría indica que utilizaría o revisaría rúbricas públicas disponibles en el LMS institucional, como una forma de aprovechar el banco de Rúbricas del TEC. De acuerdo con el área de Capacitación Interna del TEC, el proceso de formación ofrecido, su contenido y el equipo de especialistas a cargo recibió, en promedio, una evaluación de más del $92 \%$ por parte de los profesores participantes. 
Cuadro II

RESULTADOS DE LA CAPACITACIÓN

\begin{tabular}{|c|c|}
\hline $\begin{array}{l}\text { Criterio } \\
\text { valoración }\end{array}$ & Resultados \\
\hline $\begin{array}{l}\text { Metodología de la ca- } \\
\text { pacitación }\end{array}$ & $\begin{array}{l}\text { El total de quienes respondieron indican que } \\
\text { los pasos propuestos en la capacitación para } \\
\text { la creación de rúbricas ayudaron a atender el } \\
\text { proceso y fueron claros. Al menos el } 60 \% \text { de } \\
\text { los participantes utilizaron las recomendaciones } \\
\text { planteadas para la definición de los niveles de la } \\
\text { rúbrica. }\end{array}$ \\
\hline $\begin{array}{l}\text { Revisión de la rúbrica } \\
\text { por un colega }\end{array}$ & $\begin{array}{l}\text { El } 100 \% \text { valora entre media-alta y alta la im- } \\
\text { portancia de esta actividad en la definición de } \\
\text { su propia rúbrica. El } 84 \% \text { mejoró su rúbrica a } \\
\text { partir de la revisión del colega, el restante } 16 \% \\
\text { corroboró o validó su rúbrica. }\end{array}$ \\
\hline Compartir rúbrica & $\begin{array}{l}\text { Al menos un } 50 \% \text { de los encuestados compar- } \\
\text { tiría su rúbrica con otros docentes. }\end{array}$ \\
\hline $\begin{array}{l}\text { Posibilidades de re- } \\
\text { utilizar rúbricas }\end{array}$ & $\begin{array}{l}\text { Al menos el } 30 \% \text { de los encuestados usarían } \\
\text { rúbricas creadas por los compañeros. Más del } \\
50 \% \text { de los encuestados usaría rúbricas públicas } \\
\text { dentro del TEC Digital. }\end{array}$ \\
\hline
\end{tabular}

\section{CONClusiones y trabajo Futuro}

El uso de esta metodología de enseñanza-aprendizaje que considera los componentes clave de la propuesta para la creación y validación de rúbricas permitió al participante definir su rúbrica de una forma incremental y confirmar el conocimiento adquirido. Por otra parte el trabajo cooperativo-colaborativo permitió adquirir conocimientos, la interiorización, la asimilación y reflexión para desarrollar actitud crítica y tomar decisiones al uso de rúbricas en el proceso educativo.

La mayoría de los docentes utilizaría rúbricas desde un repositorio institucional, donde estas se encuentren públicas y pueden ser consultadas para su uso desde el LMS. Así como, casi una tercera parte valora utilizar rúbricas de otros docentes. Las capacitaciones y su contenido son aceptados y valorados positivamente por los docentes participantes, incluso recomiendan que otros docentes se capaciten. El ahorro en tiempo para realizar las marcas en las rúbricas y la facilidad en la devolución de resultados a los estudiantes son aspectos que motivan a los docentes a invertir trabajo en la construcción de estos instrumentos no solo por lo que indican expertos [14] y [11] sino también porque los participantes lo vivieron en las pruebas realizadas en la sesión 5 .

En el futuro y para comprobar el efecto de este proceso de formación docente se puede enfocar el seguimiento en las siguientes acciones:

1. Utilización de las rúbricas a lo largo de distintos periodos lectivos.

2. Fortalecimiento del Banco de Rúbricas, dar seguimiento a un modelo de revisión y mantenibilidad soportado en un grupo de expertos trabajando de forma cooperativacolaborativa.

3. Creación de nuevas rúbricas por parte de los profesores participantes en la formación, como una forma de comprobar que adquirieron las capacidades para este efecto.
4. Generación de indicadores que permitan validar la calidad de la rúbrica utilizando diferentes variables, tales como: opinión del evaluado, resultados de la evaluación, frecuencia de uso, resultados de mejora en las categorías evaluadas.

5. Realizar investigación educativa respecto al uso de rúbricas integradas en el LMS desde la perspectiva de profesores y estudiantes, tomando como referencia los factores propuestos por [14].

\section{REFERENCIAS}

[1] M. Raposo and E. Martínez, "La rúbrica en la enseñanza universitaria: un recurso para la tutoría de grupos de estudiantes," Formación universitaria, vol. 4, no. 4, pp. 19-28, 2011.

[2] M. Ibarra, e-Evaluación orientada al e-Aprendizaje estratégico en Educación Superior. Narcea Ediciones, 2011, vol. 29.

[3] M. Raposo, M. Rivas, and E. Martínez-Figueira, "Electronic rubrics to assess competences in ict subjects," European Educational research journal, vol. 13, no. 5, pp. 584-594, 2014.

[4] C. A. Mertler, "Designing scoring rubrics for your classroom," 2001.

[5] C. Gautreau, "Motivational factors affecting the integration of a learning management system by faculty." Journal of Educators Online, vol. 8, no. 1, p. n1, 2011.

[6] A. Mugarra, "Tics y competencias tecnológicas en el marco de bolonia: experiencias para su generación en el ámbito docente," Avances en la innovación universitaria: Tejiendo el compromiso de las universidades, pp. 731-743, 2012.

[7] A. Jones, "A review of the research literature on barriers to the uptake of ict by teachers." 2004.

[8] J. Espinoza, P. Gamboa, A. Francesa, and M. Chacǿn, "Rúbricas herramienta de impacto en la calidad de la enseñanza aprendizaje," in Tecnología y Aprendizaje. Avances en el Mundo Académico Hispano, S. y. Z. A. Prieto, M. ; Pech, Ed. Ciudad Real, España: Editorial Comunidad Internacional para el Avance de la Tecnología en el Aprendizaje, 2017 in press.

[9] F. Gatica-Lara and T. d. N. J. Uribarren-Berrueta, “¿ cómo elaborar una rúbrica?" Investigación en educación médica, vol. 2, no. 5, pp. 61-65, 2013.

[10] V. Marın, J. Cabero, and J. Barroso, "La rúbrica de evaluación en el proceso de formación del docente universitario. la propuesta del proyecto dipro 2.0," Educar, vol. 48, no. 2, pp. 347-364, 2012.

[11] D. D. Stevens and A. J. Levi, Introduction to rubrics: An assessment tool to save grading time, convey effective feedback, and promote student learning. Stylus Publishing, LLC, 2013.

[12] V. Gámiz-Sánchez, N. Torres-Hernández, and M. J. Gallego-Arrufat, "Construcción colaborativa de una e-rúbrica para la autoevaluación formativa en estudios universitarios de pedagogía," 2015.

[13] J. J. Torres Gordillo and V. H. Perera Rodríguez, "La rúbrica como instrumento pedagógico para la tutorización y evaluación de los aprendizajes en el foro online en educación superior," Pixel-Bit ( $N^{o} 36, p$. 141-149), 2010

[14] D. Atkinson and S. L. Lim, "Improving assessment processes in higher education: Student and teacher perceptions of the effectiveness of a rubric embedded in a 1ms," Australasian Journal of Educational Technology, vol. 29, no. 5, 2013.

[15] R. E. Slavin, Aprendizaje cooperativo: teoría, investigación y práctica.

[16] R. M. Gillies, A. F. Ashman, and J. Terwel, "The teacher's role in implementing cooperative learning in the classroom: An introduction," The Teacher's Role in Implementing Cooperative Learning in the Classroom, p. 1.

[17] A. Ontoria, A. Ballesteros, M. C. Cuevas, L. Giraldo, I. Martín, A. Molina, A. Rodríguez, and U. Vélez, Cómo ordenar el conocimiento: usando mapas conceptuales. Alfaomega, 2004.

[18] L. Anglin, K. Anglin, P. L. Schumann, and J. A. Kaliski, "Improving the efficiency and effectiveness of grading through the use of computerassisted grading rubrics," Decision Sciences Journal of Innovative Education, vol. 6, no. 1, pp. 51-73, 2008. 